Communications in Physics, Vol. 24, No. 3S1 (2014), pp. 127-135

DOI:10.15625/0868-3166/24/3S1/5463

\title{
A FIRST PRINCIPLES STUDY ON ELECTRONIC AND MAGNETIC PROPERTIES OF DEFECTS IN ZnO/GaN CORE-SHELL NANOWIRE HETEROSTRUCTURES
}

\author{
LE THI HONG LIEN, VU NGOC TUOC, NGUYEN VIET MINH \\ Institute of Engineering Physics, Hanoi University of Science and Technology \\ TRAN DOAN HUAN \\ Institute of Engineering Physics, Hanoi University of Science and Technology \\ and \\ Institute of Materials Science, University of Connecticut, CT 06269-3136, USA \\ E-mail: lien.lethihong@hust.edu.vn
}

Received 20 June 2014

Accepted for publication 20 August 2014

\begin{abstract}
To date semiconductor nanowire $(N W)$ heterostructures $(H S)$ have attracted extensive attention as important components of electronic and optoelectronic nanodevices. Further NWs also show promising potency to enhance the solar energy harvesting, e.g. improving both light trapping, photo-carrier collection, and contacting surface area. In this work we show theoretically that the $d^{0}$-ferromagnetism and NW HS bandgap can be turned by engineering the $H S$ interfaces in non-magnetic ZnO/GaN core/shell NW HS. In that NW HS the incorporation of one compound into the other leads to the bandgap narrowing in the nonisovalent alloy because of the type II band alignment betwwen $\mathrm{ZnO}$ and $\mathrm{GaN}$. The $d^{0}$-ferromagnetic interface can be developed by creating p-type defect with $N$ and/or $n$-type defect with $\mathrm{Zn}$ in $\mathrm{Ga}-\mathrm{O}$ interface bonds due to the defect-induced polar discontinuity. It's noted that the GaN/ZnO NW HS itself without defect or with same number defects of both types are not ferromagnetic. So that the induced magnetic moment is suggested to be related to the missing charge introduced at these defects. In our study we focused on the effects of GaN/ZnO interfaces on the electronic and magnetic properties, e.g. interface states within the bandgap and interface-induced ferromagnetism and impact of surface reconstruction and quantum confinement. The origin of this $d^{0}-F M$ is revealed by analyses of spin-polarized bandstructure indicated by the asymmetrical spin-up and spindown states near the Fermi level, the projected densities of states (PDOSs) and the spin-polarized mulliken charge differences, indicated that most spin-polarized states are dominated by the interface defect site Np electrons. The calculated GaN/ZnO interface magnetism, have been compared with $\mathrm{FM}$ at the $\mathrm{LaAlO}_{3}-\mathrm{SrTiO}_{3}$ interface which are theoretically predicted [30] and experimentally confirmed [31], where the magnetic moments also arise from the polar discontinuity.
\end{abstract}

Keywords: density functional theory, $d^{0}$-ferromagnetism, heterostucture interface, core/shell nanowires.

\section{INTRODUCTION}

Semiconductor hetero-nanostructures, well known as one of the most important components of electronic and optoelectronic nanodevices, have attracted extensive attention [1-4]. Todate by engineering the interfaces in the nano-heterostructures, nanodevices with versatile functions can be realized [5-9]. In hetero-nanostructures materials, the reconstruction of charge and 
spin at the interface can strongly affect the microscopic properties of the structure. Nanomaterials with diverse functions can be achieved by precisely controlling the atomic structure and composition at the interface. Moreover, physical properties of a nanostructure are still dramatically affected by its surface reconstruction and quantum confinement.

Semiconductor core/shell hetero-structured (HS) nanowires (NWs), of which a core and a shell are made of different material, are promising for electronic and optoelec-tronic device applications, e.g., high-efficient, multicolor light emitting diodes [1,2], solar cells [3], lasers [4]. Experimental works [1-9] and theoretical works [10-15] indicate that various physical properties of these structures, especially the optical properties, can be tuned by several experimentally attainable parameters, including the size of the core and the shell. This is one of the compelling advantages which fuel the broad interest on the structures. For a nano semiconductor structure, the surface reconstruction induced by the dangling bonds on the surface is another essentially important and dramatically affects the structural and the electronic properties [16-21]. In case of a $\mathrm{ZnO} / \mathrm{GaN}$ core/shell $\mathrm{NW}$, the core/shell interface is another important factor. In addition, the quantum confinement in the HS NW is related to both the surface and the core/shell interface because the $\mathrm{ZnO} / \mathrm{GaN}$ core/shell interface forms a radial heterostructure of type II [22,23] which leads to the bandgap narrowing in this nonisovalent alloy. Thus for the carriers which are already confined by the surface, residing inside the interface is favorable. Both $\mathrm{GaN}$ and $\mathrm{ZnO}$ are direct wide bandgap semiconductors and have been used in optoelec-tronics. GaN is often used as the substrate for the growth of $\mathrm{ZnO}$, and vice versa, because they have the same wurtzite crystal structure and lower lattice mismatch $(1.8 \%)$. All of these factors, simultaneously existing in the $\mathrm{ZnO} / \mathrm{GaN}$ core/shell HS NWs, result in combined effects on the properties of the structure. Recent development on the growth techniques, enables the growth of high quality of GaN/ZnO nanomaterials in a controlled manner [23,24]. The mechanism of the bandgap narrowing has been extensively debated and reported to attribute to the strong short-range order of $\mathrm{GaN}$ and $\mathrm{ZnO}$ in the alloy [25].

We present in this paper, a study on the electronic and magnetic properties of the interface defects in $\mathrm{ZnO} / \mathrm{GaN}$ core-shell HS NWs. We found that the core/shell HS NWs $\mathrm{ZnO} / \mathrm{GaN}$ with some specific inteface's defects could be be ferromagnetic. Further the interplay between surface and interface effect may improve the charge-separation at the core-shell interface as well as narrowing the bandgap, leading to the technological application of visible-light absorption and/or photocatalyst.

This paper is organized as follows. In Sec. II, we describe the core/shell HS NWs and a reference model in which the surface effects are suppressed. Details on the simulation method used in this works are then introduced in Sec. III. Sec. IV we discuss the obtained results for the structural, electronic and magnetic properties of the studied NWs. The paper is closed by Sec. V with some conclusions.

\section{ZnO/GaN CORE/SHELL NWs AND THEIR PASSIVATED MODEL}

The building block of the $\mathrm{ZnO} / \mathrm{GaN}$ core/shell NWs studied in this work is a hexagonal base layer, or simply the base layer, which is illustrated in Fig. 1. The base layer of a NW can be thought as being composed of several hexagonal rings of atoms enclosing the NW's axis, taken to be parallel to the $z$-axis. The inner $\mathrm{ZnO}$ rings are called the core rings while the other rings, made of $\mathrm{GaN}$, are called the shell rings. We consider three series of $\mathrm{ZnO} / \mathrm{GaN}$ core/shell $\mathrm{NWs}$ 
of hexagonal cros-sectional shape, each of them has several NWs and with the different doping content. These series are labeled by shell-core ratio of which referred by the number of the lateral hexagonal blocks at each hexagonal side as NW31, NW32 and NW42. For an illustration see Fig. 1 and its caption in details.
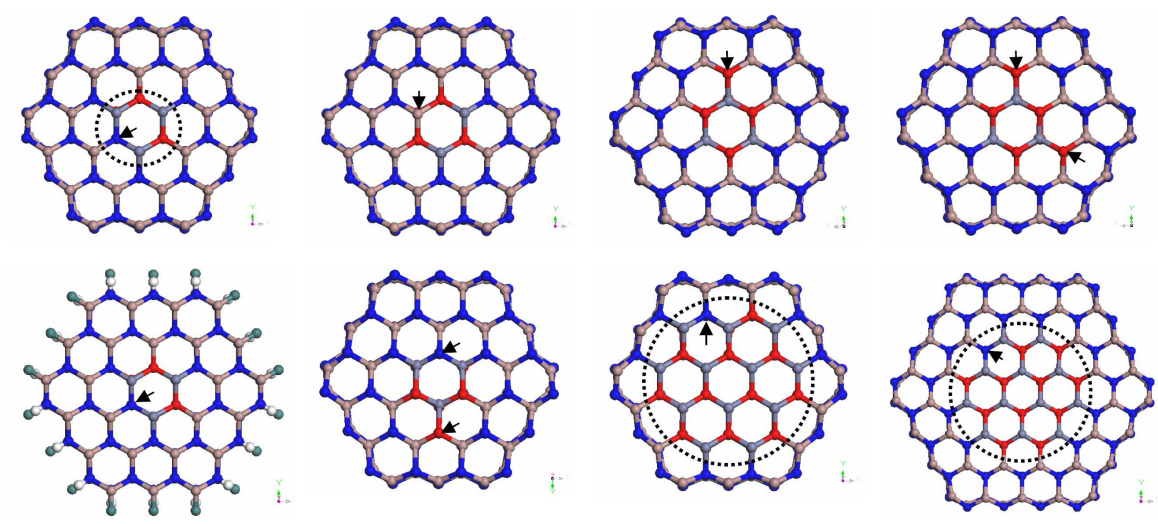

Fig. 1. Several examples of the unpassivated and passivated core/shell $\mathrm{HS} \mathrm{NWs} \mathrm{ZnO} / \mathrm{GaN}$ and the examined interface's defect positions (shown by black arrow). Zinc and oxygen atoms are shown by light-blue-gray and red spheres, where as galium and nitrogen by light yellow and blue sphere. Pseudohydrogen atoms charged by $1.25 \mathrm{e}$ and $0.75 \mathrm{e}$ are shown in green and white, respectively. Interface boundary show by dashed black circles.

It should be mention that for a NW, the surface reconstruction is an important factor that strongly affects physical properties of the NWs. To extract from other effects e.g. interface and doping, and estimate the effects from the surface reconstruction, for each NW, we additionally study a reference NW in which a number of pseudo hydrogen atoms are used to saturate the surface dangling bonds, thus suppressing the surface reconstruction. We call the reference NWs by passivated NWs ans refer to as NWP, while the original NWs are called unpassivated and refer to as NW. Thus the deviation of the physical properties of an unpassivated NW from those of the corresponding passivated NW is assumed to be resulted by the surface reconstruction. For more detail discussion of using the pseudo hydrogen and passivated NW model, see Refs. [21,26].

To investigate the effects of the interfaces on the electronic and magnetic properties of the core/shell HS NWs, various possible interfaces defects are considered. At the Ga-O interface, this include $\mathrm{N}$-substitution of $\mathrm{O}$ (denote as $\left.\mathrm{N}_{O}\right)$, Zn substitution of $\mathrm{Ga}\left(\mathrm{Zn}_{G A}\right)$. The defects at the $\mathrm{Zn}-\mathrm{N}$ interface include $\mathrm{O}_{N}, \mathrm{Ga}_{Z n}$. We also studied the defect pairs at both of the interfaces, i.e. including together $\mathrm{N}_{O}$ and $\mathrm{O}_{N}$, double $\mathrm{N}_{O}$ and double $\mathrm{O}_{N}$.

\section{COMPUTATIONAL METHOD AND DETAILS}

We use in this work the version of density functional theory (DFT) implemented in SIESTA (Spanish Initiative for Electronic Simulations with Thousands of Atoms), the electronic structure code that implements linear-scaling methods for gapped systems $[27,28]$. The flexible localized numerical double-zeta-plus polarization basis set is used, allowing arbitrary angular momenta, multiple radial functions per angular momentum, and polarized and off-site orbitals [28]. Some 
figures in this work are prepared by XCrySDen [29]. Norm-conserving Troullier-Martins nonlocal pseudo-potentials with partial core corrections are used for our calculations. The particular valence electron configurations of zinc, oxygen, nitrogen and gallium are $3 d 104 s 2,2 s 22 p 4$, $2 s 22 p 3$, and $4 s 23 d 104 p 1$ respec-tively. Of these configurations, the Ga-3d and Zn- $3 d$ electron states are treated explicitly as valence states to describe the hybridization between the $\mathrm{Ga} / \mathrm{Zn}-3 d$ and N/O- $2 p$ orbitals.

The pseudopotentials for the pseudo hydrogen atoms charged $1.25 \mathrm{e}$ and $0.75 \mathrm{e}$ are generated from the pseudopotential of ordinary hydrogen atom using the "fractional" program implemented in Virtual Crystal Approximation, a tool provided by SIESTA 3.x, in which the pseudo hydrogen atoms are treated as the "synthesized" atoms. The Perdew-Burke-Ernzerhof (PBE) functional is used for exchange correlation energy of all the species. The initial structures of the NWs are designed directly from the lattice of the bulk GaN material and then corresponding atoms in core are replaced by zinc and oxygen atoms. For a nanowire, the simulation box contains two base atomic layers wrapped by at least $10 \AA$ of vacuum thickness along the $x$ and $y$ directions to eliminate the interaction with its periodical images. Along the $z$ direction, we employed the periodic boundary condition to capture the quasi one-dimensional (Q1D) nature of the nanowires. The lattice parameter $\mathrm{c}$ was determined by minimizing the total energy of the system with respect to $c$ - lattice parameter along $z$ direction. For the passivated and unpassivated nanowires, we found $c=5.28 \AA$ and $c=5.33 \AA$, respectively. The structures were then optimized until the forces exerting on the atomic sites are less than $0.01 \mathrm{eV} / \AA$. The convergences of the total energy and the optimized geometries were tested with respect to the size of the basis set and the dimensions of the simulation box.

\section{RESULTS AND DISCUSSIONS}

From the optimized structure obtained in the previous Section, we have calculated the band structures of the NWs. In Fig. 2, the band structure of the passivated together with their unpassivated are shown. Comparing to those of the passivated, the conduction band and the valence band of the unpassivated are reconstructed with some new surface-induced states residing around the valence band maximum (VBM) - (marked by red in Fig. 2). In addition it is clearly that eliminating these surface-induced states, the two bands of undoped NW and NWP, which are tailoring side-by-side at Fig. 2. left-half are nearly identical upto some shifting constants. As a consequence of the surface-induced states, the band gap of the unpassivatedis dramatically reduced in both with and without inteface doping cases, as seen from Fig. 2. left-right.

Next, we show in Fig. 3 the spin-up and spin-down energy level structures at the $\Gamma$ point of several passivated and unpassivated NW with various type of doping. With this we can conclude that, defect-free $\mathrm{ZnO}$ nanowires exhibit no magnetic moment since the band structures of $\mathrm{NW}$ and NWP are constructed by pairs of identical spin-up and spin-down bands. Therefore, the total occupied spin-up states and the total occupied spin-down states are equal, thus the magnetic moment of either NW or NWP, is $M \equiv 0 \mu_{B}$. Similarly reason, for the case of NWs with such defects as $\mathrm{O}_{N}, \mathrm{Ga}_{Z n}$ and $\mathrm{O}_{N}+\mathrm{N}_{O}$. In other kind of defects, e.g. $\mathrm{N}_{O}$ all the spin-down bands locating at up to the highest acceptor level (a few $0.1 \mathrm{eV}$ above the Fermi level) are systematically shifted up from the corresponding spin-up bands (see Fig. 2 right-half and also Fig. 3 column $3^{\text {rd }}$ and $4^{\text {th }}$ ) results in magnetic moment $M=1.0 \mu_{B}>0$. On the other hand, the systems of spin-up and spin-down levels in the conduction bands of the NWs are nearly identical. Consequently, this band structure 

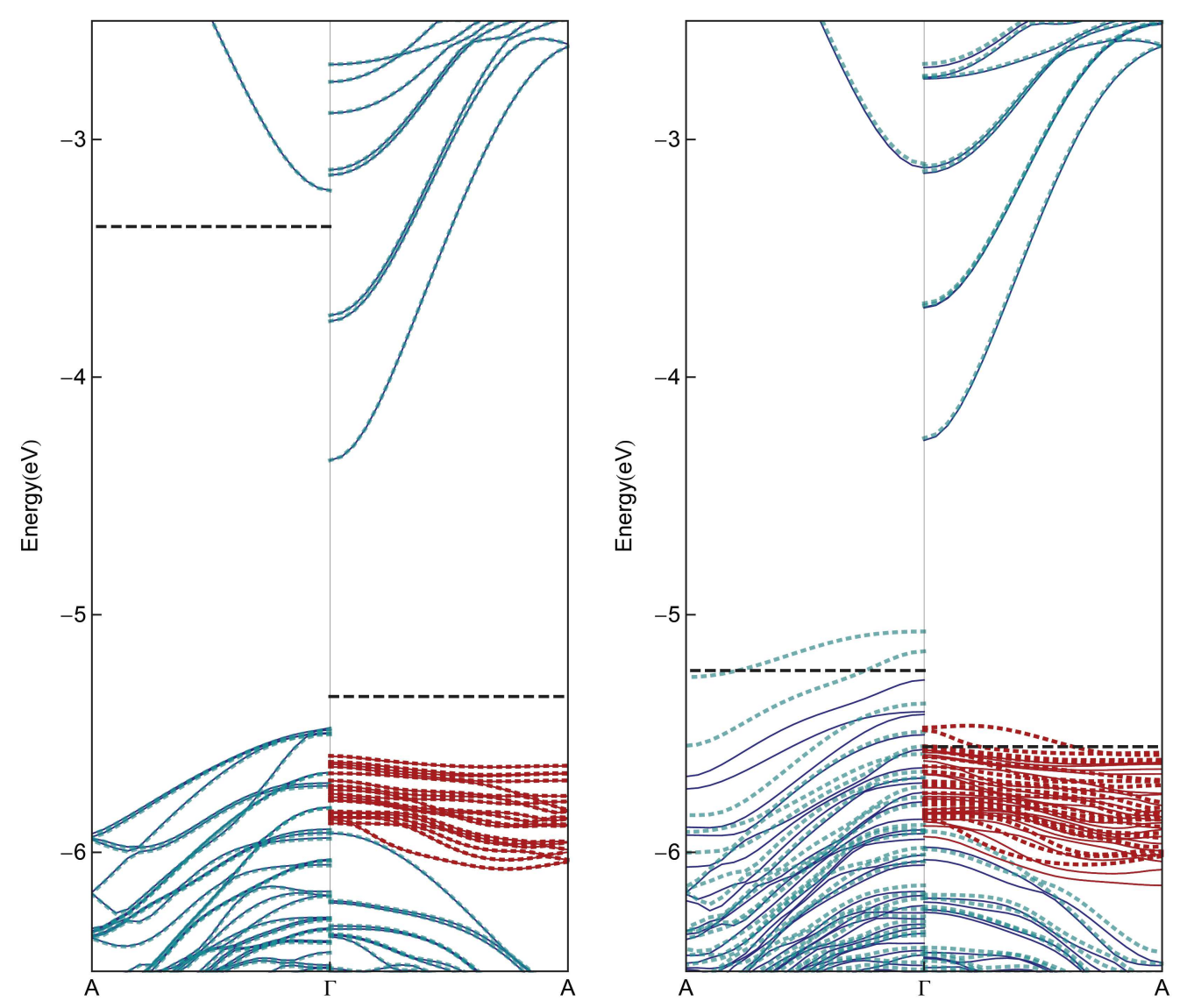

Fig. 2. Band structures of undoped core/shell NW31-NWP31 (left) and NW31 $\mathrm{N}_{O^{-}}$ NWP31N $O$ (right). Spin-up and spin-down bands are shown by solid and dashed curves (note that for undoped NW any spin-up band, there is an identical spin-down band). Surface-dominated states of NW are shown in red.

is signaling the hole-induced ferromagnetism of the interface-defect's NW. Although the surface reconstruction does not induce FM in defect-free $\mathrm{ZnO} N W$, it might tune the magnetic moment $M$ of the $\mathrm{ZnO}$ nanowires with defects.

The calculated Projected Densities of States (PDOSs) of the core/shell HS NWs ZnO/GaN with defects at the $\mathrm{Ga}-\mathrm{O}$ interface $\left(\mathrm{Zn}_{G a}\right.$, and $\left.\mathrm{N}_{O}\right)$ clearly demonstrate their ferromagnetism (Fig. $4 \mathrm{a}, 4 \mathrm{e})$, as indicated by the asymmetrical spin-up and spin-down states near the Fermi level. The holes in the spin-down structure are strongly spin-polarized, leading to its ferromagnetism. Further analysis on PDOS indicates that the spin-polarized states are dominated by the N-p electrons at the $\mathrm{Zn}-\mathrm{N}$ interface bonds (fig.4a), with the solid thick line for spin-up and the thick dashed for spin-down. The calculated magnetic moment with this structure is $\sim 1.0 \mu_{B}$. We can also see that there some localized states are within the band gap of both the spin-up and spin-down states (at about $1.4 \mathrm{eV}$ above the Fermi level), which may reduce the bandgap) marked by black arrow. Further observation implies that the electrons in the conduction band make very small 


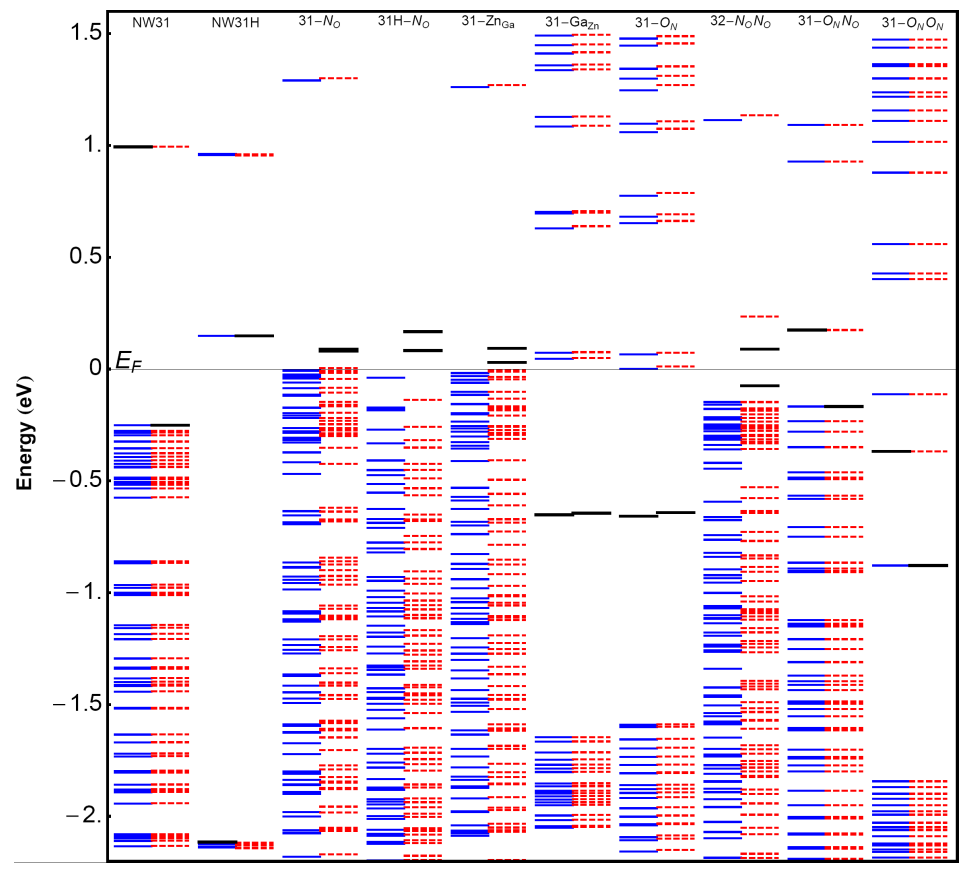

Fig. 3. $\Gamma$-point band structures of various passivated and unpassivated undoped and doped NWs. Fermi energies $E_{F}$ are shifted to zero. Spin-up and spin-down states are indicated by solid and dashed lines. Most of the states below the Fermi level shown in this figure are surface-dominated. Highest occupied molecular orbital (HOMO) and lowest unoccupied molecular orbital (LUMO) are given as bold solid lines.

or no contribution to the magnetic moment $M$ (see Fig. 4). With the behavior up to the Fermi level as there are more spin-up electrons than the spin-down electrons, therefore, for in this case dopings are able to induce FM in HS NWs. To clarify the mechanism of the $\mathrm{ZnO} / \mathrm{GaN}$ HS NW interface's magnetism in the case, the comparison can be made to $\mathrm{LaAlO}_{3}-\mathrm{SrTiO}_{3}$ interface of their layer structure. There oxide hetero-interface introduces polar discontinuities, resulting in different insulating $p$-type $(\mathrm{SrO})-(\mathrm{AlO} 2)^{-}$and conducting $n$-type $(\mathrm{TiO} 2)-(\mathrm{LaO})^{+}$interface $[30$, 31]. There the ferromagnetic alignment of the induced electron spins within the $n$-type (TiO2)$(\mathrm{LaO})^{+}$interface had been theoretically predicted [30] and experimentally confirmed [31], where the magnetic moments is shown to arise from the polar discontinuity.

Similarly here on the case that Ga-N bond shows stronger charge-polarization than $\mathrm{Zn}$ $\mathrm{O}$ one as it shows from Fig. 6 (a) and (b) with full spin-polarized mulliken charges per site are given ( $\sim 0.8 e$ vs $0.56 e$, with $e$ is electron charge). So that in pure NWs the N- or O-terminated surface is negatively charged, while Ga- or Zn-terminated surface is positively charged. However, for the $\mathrm{GaN} / \mathrm{ZnO}$ core/shell heterostructure $\mathrm{NW}$, with the presence of hetero-interfaces, the $\mathrm{Zn}-\mathrm{N}$ interface bond is $p$-type (because of $\mathrm{N}$ has is less valence electron than $\mathrm{O}$ does), and the $\mathrm{Ga}-\mathrm{O}$ interface bond is $n$-type (see more in Fig. 6a. and 6b.) as already discussed in [32]. Our conclusion that the $p$-type $\mathrm{Zn}-\mathrm{N}$ interface defect bonds gives rise to FM ordering and it is mainly contributed from the N-p electrons at the interface defect site. 

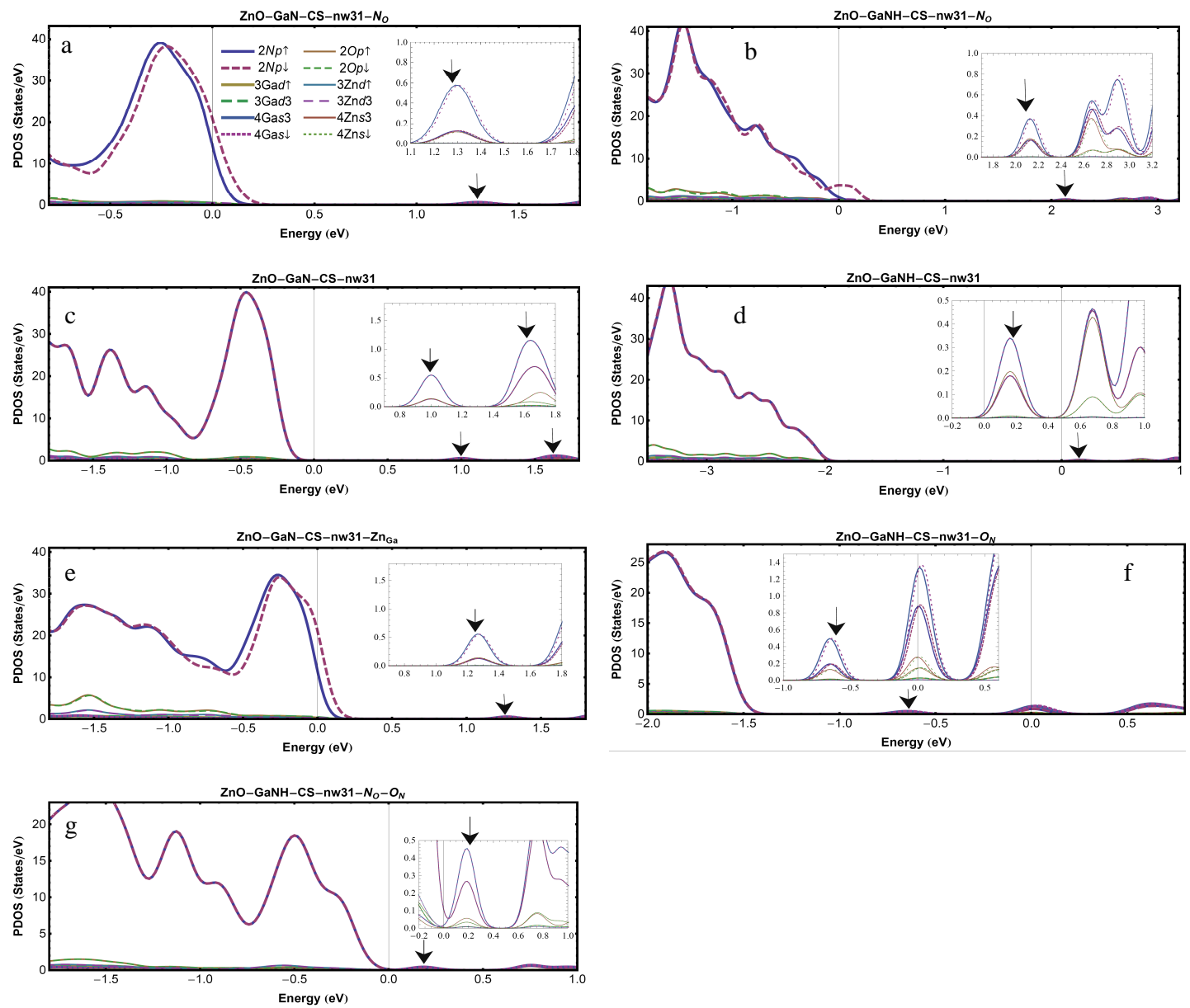

Fig. 4. Density of electron states projected on atomic orbitals of various passivated and unpassivated NWs with and without doping. Only dominant contributions are shown. Fermi energies were set to zero.

However different from the case of $\mathrm{LaAlO}_{3}-\mathrm{SrTiO}_{3}$, where the FM order come from conducting electron, i.e. two-dimensional (2D) spin-polarized electron gas at the interface $n$-type ( $\mathrm{TiO} 2)-(\mathrm{LaO})$ conducting interface [31], herein $\mathrm{ZnO} / \mathrm{GaN}$ core/shell $\mathrm{NW}$ it is $p$-type, i.e. holeinduced FM, see also the spin-polarized band structure discussed above. Because the FM ordering come from $\mathrm{Zn}-\mathrm{N}$ interface's defect bonds so in $\mathrm{ZnO} / \mathrm{GaN}$ core/shell $\mathrm{NW}$ without defects and with defects including $\mathrm{Ga}_{Z n}, \mathrm{O}_{N}$ the magnetic moment equal zero. For the case of dual defect $\mathrm{N}_{O}+\mathrm{O}_{N}$ we also get $M=0 \mu_{B}$. The above observations clearly suggest a steady connection between the total number of missing valence electrons of a defect, the local structural reconstruction surrounding the defect, and the defect-induced local magnetic moment $M_{l o c}$. This connection is able to provide an explanation for the enhancement of the FM by two defect (e.g. $\mathrm{N}_{O}+\mathrm{N}_{O}$ ) or absent of $M$ in case of $\mathrm{O}_{N}+\mathrm{N}_{O}$ as described above. 
Fig. $6(c, d, e, f)$ shows spin-polarized Mulliken charge difference between site's spin-up and spin-down states in the plane that contains defect. It reveals the localization of the spin-polarization effect for the interface defects NWs. The local atom magnetic moment is maximum at the nitrogen defect's site (Fig. 6c and d) and are quickly close to zero while moving from defect's site. This can also be clear in Fig. 5 showing the isosurface of the spin difference density of unpassivated with interface doping defect $\mathrm{N}_{O}$ (fig.5a) and its passivated reference (Fig. 5b). Further compare on Fig. 6 (c) and (d), illustrates that the local magnetic moment $M_{l o c}$ of an $N$-defect atom, is affected by the dangling bond at the outer surface, i.e. the NW surface reconstruction. Fig.6e show the case of $\mathrm{NWP} 32 \mathrm{~N}_{O}$, comparing with Fig. $6 \mathrm{~d}$ proves that with the passivation scheme, the surface reconstruction is suppressed and has no effect on the local magnetic moment of the $N$-defect site so that although the core/shell interfaces are different, i.e. NWP31 $\mathrm{N}_{O}$ and $\mathrm{NWP} 32 \mathrm{~N}_{O}$, the magnetic moments are the same. (see Fig. $6 \mathrm{~d}$ and $6 \mathrm{e}$ for an illustration). In fig.6f we show the spatial distribution of $M_{l o c}$ on the case NWP42 $\mathrm{N}_{O}-\mathrm{N}_{O}$ that contains double defects. It shows that magnetic moment not only depend on the total number of missing valence electrons at $N$-defect sites but still on the location of defect from each to other, and there might have some saturation effect with the increasing defect sites. It may due to the fact that around a defect site, e.g. a $\mathrm{N}_{O}$ or $\mathrm{Zn}_{G a}$, there is a local structural reconstruction which is driven by the dangling bonds at the defect's site starting from the atoms nearby.

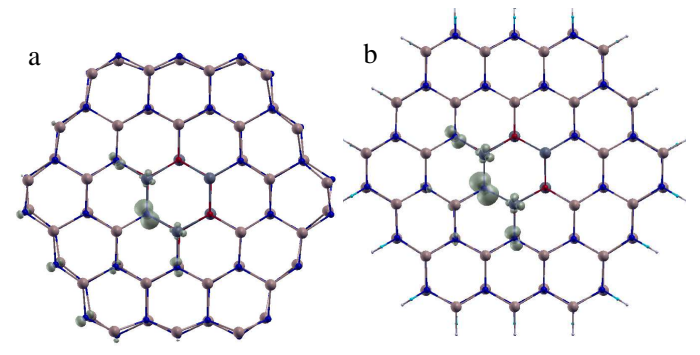

Fig. 5. The iso-surface of the spin difference density of unpassivated $\mathrm{NW} 31 \mathrm{~N}_{O}$ (left) and passivated (right) NWP31 $\mathrm{N}_{O}$.
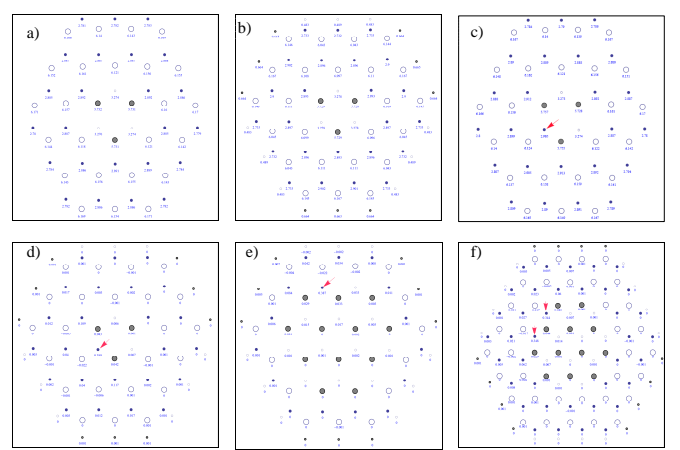

Fig. 6. Spinpolarized Mulliken charge of the undoped (a) unpassivated nanowire NW31 and (b) passivated NWP31; Spin-polarized Mulliken charge difference for various doping case (c) $\mathrm{NW} 31 \mathrm{~N}_{O}$, (d) $\mathrm{NWP} 31 \mathrm{~N}_{O}$, (e) NWP $32 \mathrm{~N}_{O}$, (f) $\mathrm{NWP} 42 \mathrm{~N}_{O}-\mathrm{N}_{O}$. The zinc and gallium atoms are shown by large filled and empty circles, oxygen and nitrogen by medium empty and filled circle whereas, pseudo hydrogen charged $1.25 \mathrm{e}$ and $0.75 \mathrm{e}$ by small filled and empty circles, doping sites are marked by the red arrow.

\section{CONCLUSIONS}

We studied in this work the ferromagnetism induced by $p$-type interface defect the core/shell HS NWs $\mathrm{ZnO} / \mathrm{GaN}$. It is clearly demonstrated that the interface defects in $\mathrm{ZnO} / \mathrm{GaN}$ nanowires generate at each of the nitrogen atoms around the defect site a given local magnetic moment and it is major contribution to HS NWs magnetic moment. The spin-polarization effect of a defect is strongly localized, implying that local magnetic moment is dominated by contributions within a closed local region, surrounding the defect. We also found that the nanowire surface has significant impacts on the induced ferromagnetism and band gap narrowing. A correlation between 
the local structural reconstruction around a defect and the magnetic moment induced was then unveiled. Calculated results from our work indicate that by using the passivated nanowire reference model, we were able to figure out the effects from the surface reconstruction on the defect-induced ferromagnetism of these HS NWs.

\section{ACKNOWLEDGMENT}

This research is funded by Vietnam National Foundation for Science and Technology Development (NAFOSTED) under grant No 103.02-2011.20.

\section{REFERENCES}

[1] F. Qian, Y. Li, S. Gradecak, D. Wang, C. J. Barrelet, and C. M. Lieber, Nano Lett. 4 (2004) 1975.

[2] F. Qian, S. Gradecak, Y. Li, C.-Y. Wen, and C. M. Lieber, Nano Lett. 5 (2005) 2287.

[3] Y. Dong, B. Tian, T. J. Kempa, and C. M. Lieber, Nano Lett. 9 (2009) 2183.

[4] S. Gradecak, F. Qian, Y. Li, H.-G. Park, and C. M. Lieber, Appl. Phys. Lett. 87 (2005) 173111.

[5] I. Arslan, A. A. Talin, and G. T. Wang, J. Phys. Chem. C 112 (2008) 11093.

[6] L. Rigutti, G. Jacopin, L. Largeau, E. Galopin, A. De Luna Bugallo, F. H. Julien, J.-C. Harmand, F. Glas, and M. Tchernycheva, Phys. Rev. B 83 (2011) 155320.

[7] V. Laneuville, F. Demangeot, R. Pechou, P. Salles, A. Ponchet, G. Jacopin, L. Rigutti, A. de Luna Bugallo, M. Tchernycheva, F. H. Julien, K. March, L. F. Zagonel, and R. Songmuang, Phys. Rev. B 83 (2011) 115417.

[8] K. Hestroffer, R. Mata, D. Camacho, C. Leclere, G. Tourbot,Y. M. Niquet, A. Cros, C. Bougerol, H. Renevier, and B. Daudin, Nanotechnology 21 (2010) 415702.

[9] R. Calarco, M. Marso, T. Richter, A. I. Aykanat, R. Meijers, Avd. Hart, T. Stoica, and H. Luth, Nano Lett. 5 (2005) 981

[10] B. Pal, K. Goto, M. Ikezawa, Y. Masumoto, P. Mohan, J.Motohisa, and T. Fukui, Appl. Phys. Lett. 93 (2008) 073105.

[11] B. Goswami, S. Pal, and P. Sarkar, J. Phys. Chem. C 112 (2008) 11630.

[12] J. Renard, R. Songmuang, G. Tourbot, C. Bougerol, B. Daudin, and B. Gayral, Phys. Rev. B 80 (2009) 121305.

[13] H. Lee and H. J. Choi, Nano Lett. 10 (2010) 2207.

[14] R. N. Musin and X. Q. Wang, Phys. Rev. B 74 (2006) 165308.

[15] L. Yang, R. N. Musin, X. Q. Wang, and M. Y. Chou, Phys. Rev. B 77 (2008) 195325.

[16] K. Rezouali, M. A. Belkhir, and J. B. Bai, J. Phys. Chem. 114 (2010) 11352.

[17] R. F. Khairutdinov, Russ. Chem. Rev. 67 (1998) 109.

[18] B. Meyer and D. Marx, Phys. Rev. B 67 (2003) 035403.

[19] N. Combe, P. Chassaing, and F. Demangeot, Phys. Rev. B 79 (2009) 045408.

[20] V. N. Tuoc, Comput. Mater. Sci. 49 (2010) S161.

[21] V. N. Tuoc, T. D. Huan and L.T.H. Lien Phys. Status Solidi B 249(6) (2012) 1241.

[22] S. Wang, L.W. Wang, Physical Review Letters 104 (2010) 065501.

[23] M.N. Huda, Y. Yan, S.-H. Wei, M.M. Al-Jassim, Physical Review B78 (2008) 195204.

[24] T. Song, J.W. Choung, J.G. Park, W. Park, J.A. Rogers, U. Paik, Advanced Materials 20 (2008) 4464.

[25] S. Wang, L.W. Wang, Physical Review Letters 104 (2010) 065501

[26] M. X. Xiao, M. Zhao, and Q. Jiang, Chem. Phys. Lett. 512 (2011) 251.

[27] P. Ordejon, E. Artacho, and J. M. Soler, Phys. Rev. B 53 (1996) R10441.

[28] J. M. Soler, E. Artacho, J. D. Gale, A. Garcia, J. Junquera, P. Ordejon, and D. Sanchez-Portal, J. Phys. Condens. Matter 14, 2745 (2002).

[29] A. Kokalj, J. Mol. Graph. Model. 17 (1999) 176. Code available from http://www.xcrysden.org/.

[30] R. Pentcheva, W. E. Pickett, Phys. Rev. B 74 (2006) 035112.

[31] A. Brinkman, M. Huijben, M. Vanzalk, J. Huijben, U. Zeitler, J. C. Maan, W. G. Van Der Wiel, G. Rijnders, D. H. A. Blank and H. Hilgenkamp, Nature Materials 6 (2007) 493.

[32] H. Pann, Y. W. Zhang, Nano Energy 1 (2012) 488. 